\title{
Recommendations for exercise adherence measures in musculoskeletal settings: a systematic review and consensus meeting (protocol)
}

\author{
Melanie A Holden ${ }^{1 *}$, Kirstie L Haywood ${ }^{2}$, Tanzila A Potia ${ }^{3}$, Melanie Gee ${ }^{4}$ and Sionnadh McLean ${ }^{3}$
}

\begin{abstract}
Background: Exercise programmes are frequently advocated for the management of musculoskeletal disorders; however, adherence is an important pre-requisite for their success. The assessment of exercise adherence requires the use of relevant and appropriate measures, but guidance for appropriate assessment does not exist. This research will identify and evaluate the quality and acceptability of all measures used to assess exercise adherence within a musculoskeletal setting, seeking to reach consensus for the most relevant and appropriate measures for application in research and/or clinical practice settings.
\end{abstract}

Methods/design: There are two key stages to the proposed research. First, a systematic review of the quality and acceptability of measures used to assess exercise adherence in musculoskeletal disorders; second, a consensus meeting. The systematic review will be conducted in two phases and reported in accordance with the Preferred Reporting Items for Systematic Reviews and Meta-Analyses (PRISMA) guidelines to ensure a robust methodology. Phase one will identify all measures that have been used to assess exercise adherence in a musculoskeletal setting. Phase two will seek to identify published and unpublished evidence of the measurement and practical properties of identified measures. Study quality will be assessed against the COnsensus-based Standards for the selection of health Measurement Instruments (COSMIN) guidelines. A shortlist of best quality measures will be produced for consideration during stage two: a meeting of relevant stakeholders in the United Kingdom during which consensus on the most relevant and appropriate measures of exercise adherence for application in research and/or clinical practice settings will be sought.

Discussion: This study will benefit clinicians who seek to evaluate patients' levels of exercise adherence and those intending to undertake research, service evaluation, or audit relating to exercise adherence in the musculoskeletal field. The findings will impact upon new research studies which aim to understand the factors that predict adherence with exercise and which test different adherence-enhancing interventions. PROSPERO reference: CRD42013006212

Keywords: Acceptability, Adherence, Consensus, Exercise, Measurement, Musculoskeletal disorders, Physical activity, Quality, Systematic review

\section{Background}

Musculoskeletal disorders, such as low back pain, shoulder disorders, and osteoarthritis, are common, with estimates suggesting an average prevalence of $38 \%$ [1], which increases markedly with age [1] and is likely to continue to rise due to the ageing population and increasingly

\footnotetext{
* Correspondence: m.holden@keele.ac.uk

${ }^{1}$ Arthritis Research UK Primary Care Centre, Keele University, Keele ST5 5BG, UK

Full list of author information is available at the end of the article
}

sedentary lifestyles [2,3]. Musculoskeletal disorders cause more functional limitations than any other group of disorders within the adult population and lead to enormous healthcare expenditure and loss of work [4].

Clinical guidelines advocate the use of exercise programmes for musculoskeletal disorders [5,6]. Exercise can encompass a wide range of interventions such as general (aerobic) exercise, specific body-region exercises for strengthening and flexibility, continuing normal physical activities, and increasing general physical activity levels [7]. 
Systematic reviews consistently show the beneficial effects of different types of exercise on key clinical outcomes such as pain, physical function, and quality of life [8-10]. Adherence, defined as "the extent to which a person's behaviour corresponds with agreed recommendations from a healthcare provider", is considered to be an important pre-requisite for the success of exercise programmes for musculoskeletal disorders [11,12]. Adhering to an exercise programme enhances its effectiveness, and patients who undertake regular physical activity may be less likely to progress to recurrent, persistent, or disabling problems $[13,14]$.

Despite its importance, adherence to clinic-based exercise protocols is often around 50\% $[15,16]$ and is usually worse for unsupervised home exercise programmes $[17,18]$. Non-adherence to exercise recommendations may negatively affect treatment effectiveness, treatment duration, efficiency of use of personnel and equipment, the therapeutic relationship, waiting times, and cost of care [19-21], and may also be responsible for nonsignificant research outcomes within a clinic-based research context [22]. Although numerous interventions exist that may potentially improve adherence to exercise for musculoskeletal disorders [7,23-25], in order to robustly test the effectiveness of these potential interventions, reliable and reproducible measures of exercise adherence are first required.

Due to the multi-dimensional nature of exercise adherence [26], including completing exercise and physical activity correctly, in different settings and at the agreed 'dose', accurate measurement of exercise adherence can be challenging. No gold standard measure of adherence exists [27]. Moreover, the most appropriate measure of adherence for one type of therapeutic exercise (for example specific body-region exercises for strengthening and flexibility) may not be appropriate to measure adherence to other types of therapeutic exercise, such as increasing general physical activity levels. Within clinical practice, more objective measures of exercise adherence, such as a diary, are underutilised [28], and in randomised controlled trials of exercise for musculoskeletal disorders measurement of adherence is either non-existent or limited by use of non-standardised instruments that capture data on only one domain of adherence [7,29].

Although a wide range of performance-based, clinicianreported and patient-reported measures of exercise adherence are available, most lack a clear theoretical underpinning $[7,30]$. In addition, there is wide variation in the use of these measures within clinical research and routine practice settings, and guidelines or consensus regarding how exercise adherence should be assessed do not exist. Agreement on the most relevant, useful and appropriate approach to assessing exercise adherence is essential if research and clinical audit is to be effective in informing both policy and clinical decision-making [31].

This study will seek to achieve a UK-based consensus on the 'best' measures of exercise adherence in terms of quality, acceptability, and usefulness to musculoskeletal research and routine clinical practice.

\section{Methods/design}

There are two key stages to the proposed research. First, a systematic review of the quality and acceptability of measures used to assess exercise adherence; second a consensus meeting of UK stakeholders. The systematic review will be conducted in two phases and reported in accordance with the Preferred Reporting Items for Systematic Reviews and Meta-Analyses (PRISMA) guidelines to ensure robust methodology [32]. Phase one will identify all measures that have been used to assess exercise adherence within a musculoskeletal setting. Phase two will seek to identify published and unpublished evidence of measurement and practical properties of identified measures of exercise adherence. The quality of developmental and evaluative studies will be assessed against the COnsensus-based Standards for the selection of health Measurement Instruments (COSMIN) guidelines $[33,34]$. A short-list of best quality measures will be produced for consideration during stage two of the study, the consensus meeting, which will inform recommendations on the 'best' measures of exercise adherence in terms of quality, acceptability, and usefulness to musculoskeletal research and routine clinical practice.

\section{Stage 1.1: Systematic review - identification of measures}

A search strategy combining title/abstract words and database subject headings relating to exercise adherence and musculoskeletal rehabilitation will be used to locate all measures used to assess exercise adherence in a musculoskeletal context (see Search strategy for phase one of the systematic review), and run in the following databases from their inception: Medline, SPORTDiscus, CINAHL Plus with Fulltext, PsycINFO, AMED, The Cochrane Library (Cochrane Database of Systematic Reviews, Cochrane Cemtral Register of Controlled Clinical Trials, Database of abstracts of reviews of effects, Health Technology Assessment Database, and the NHS economic evaluation database), Embase, and Web of Science. A RefWorks database will be used to manage all references.

\section{Search strategy for phase one of the systematic review}

The search strategies will use title and abstract words/ synonyms and database subject headings (e.g., $\mathrm{MeSH}$ ) to capture the concept of exercise adherence in the context of musculoskeletal rehabilitation, for adult patients. The strategies will also contain the following exclusions: 
"cardiac rehabilitation", "pulmonary rehabilitation", "neuro" rehabilitation", "stroke", "child", and "infan"”.

To capture 'exercise adherence', title and abstract terms/ synonyms and database subject headings for adherence will be combined with those for exercise (listed below) using proximity operators (within 3 words), or AND, as applicable.

Adherence terms: Words in title/abstract: adher*, nonadher*, complian $^{*}$, noncomplian ${ }^{*}$, concordan*, cooperat*, co-operat*, uncooperat*, unco-operat*, engag*, disengag*, behaviour\#, behavior\#. MeSH: "Patient Compliance".

Exercise terms: Words in title/abstract: activ*, exercis*, physical n3 train*, weight n3 train*, sport\#, rehab*, MeSH: "Therapeutic Exercise +", "Exercise Therapy +", "Exercise +", "Physical Activity", "Motor Activity", "Exercise Movement Techniques".

To restrict the results to the context of musculoskeletal rehabilitation, the above searches will be combined (AND) with the following search terms/synonyms and database subject headings:

Musculoskeletal rehabilitation terms: Words in title/ abstract: osteopath*, chiropract", musculoskeletal, msk, physiotherap*, rehabilitat*, osteoarthrit", spondyl*, osteitis, osteochondritis, arthropathy, bursitis, shoulder impingement, myalgia, lordosis, sacroiliac, sciatica, cervicogenic, dyskinesis, tendinitis, tendinopathy, allodynia, hyperalgesia, subluxation, disc, misalignment, osteopathic lesion, frozen shoulder, degenerative joint disease, muscular n3 pain, back $n 3$ pain, lumbar $n 3$ pain, lumbo* $n 3$ pain, spine $n 3$ pain, spinal $\mathrm{n} 3$ pain, neck $\mathrm{n} 3$ pain, cervical $\mathrm{n} 3$ pain, knee* $\mathrm{n} 3$ pain, hips $\mathrm{n} 3$ pain, hip $\mathrm{n} 3$ pain, shoulder $\mathrm{n} 3$ pain, ankle\# n3 pain, foot $n 3$ pain, feet $n 3$ pain, elbow\# $n 3$ pain, hand\# n3 pain, flank pain, buttock pain, joint pain, radicular pain, neuralgia, lumbago, arthralgia, adverse neural tension, muscle tear\#, sprain* n5 musc ${ }^{*}$, strain* n5 musc*. MesH: "Osteopathy", "Osteopathic Medicine", "Chiropractic", "Manipulation, Chiropractic", "Musculoskeletal Diseases +", "Sciatica”, “Tendinopathy +", “Allodynia”, "Hyperalgesia", "Subluxation", "Back Pain +", "Neck Pain”, "Neuralgia +", "Elbow Pain", "Arthralgia +", ("Musculoskeletal System +" AND "Pain +").

Titles and abstracts of all identified articles will be reviewed for inclusion by two independent reviewers and agreement checked. A third independent reviewer will be used to resolve any differences regarding eligibility. Any primary quantitative study (including clinical trials, observational studies, longitudinal studies, case control studies, and case studies) will be included if they involve: i) adults with musculoskeletal disorders, ii) any therapeutic exercise or physical activity intervention, iii) clearly defined and reproducible measures used to assessed adherence to exercise or activity, including patient-reported or clinician-reported measures or exercise diaries (if converted to an adherence measurement scale), and iv) exercise or activity delivered in any therapeutic setting including inpatient, outpatient, and community settings. Studies will be excluded if they are not written in English, involve i) participants under 18 years of age, ii) participants who have received therapeutic exercise and activity for non-musculoskeletal conditions such as diabetes, asthma, and cancer, or iii) if they include healthy volunteers. Performance measures (i.e., muscle strength and joint range of movement), performance of exercise technique and attendance at sessions are often considered proxy measures of exercise adherence and will therefore be excluded from this review. Following title and abstract screening, full text articles of retained studies will be reviewed for inclusion and adherence measures will be identified. Cleary defined and reproducible measures of exercise adherence used within the musculoskeletal field will then be located and collated.

\section{Stage 1.2: Systematic review - identification of development and/or evaluative papers}

Articles reporting the measurement and/or practical properties of identified measures used to assess exercise adherence will be sought by performing further specific searches in the databases identified above for each identified measure. A highly sensitive search filter developed by Terwee et al. [35] for finding studies on measurement properties of measurement instruments will be applied for larger result sets. Authors identified as the first contact for the development of a specific measure will be contacted to locate additional published or unpublished studies that may provide additional evidence of measurement and/or practical properties for the specific measure. Published articles will be included if they provide evidence of development and/or evaluations for clearly defined and reproducible measures used to assess exercise adherence, and published in the English language. We will seek evidence from both within, and outside of the musculoskeletal setting. All titles and abstracts, and where applicable full text articles, will be assessed for inclusion by two independent reviewers and a third reviewer will resolve any disagreements. Reference lists of included articles will be reviewed for additional published articles.

\section{Data extraction and appraisal}

A data extraction form informed by earlier reviews [36] and the requirements of the COSMIN checklist [33,34] will be used to ensure that data necessary to support an evaluation of both study and measure quality is extracted.

Data extraction will capture study-specific information (including population, intervention, and setting) and measurement tool-specific information. Extraction for 
measurement properties will seek evidence of reliability (internal consistency, test-retest, intra- or inter-tester, measurement error); validity (content, construct including internal (within scale) analyses and analyses against external criteria, convergent/divergent, and known group differences); evidence of explicit hypothesis testing will be detailed; evidence of the conceptual underpinning and the aspects of exercise adherence which the measure purports to assess will be sought; responsiveness (criterion-based or construct-based assessment); interpretation (minimal important difference); and precision (data quality, end effects). Extraction for evidence of practical properties will include acceptability (relevance and respondent burden) and feasibility. The extent of patient involvement in measurement development and/or application will also be sought.

In accordance with the COSMIN checklist, each measurement property reported by the study will be rated on a 4-point scale (i.e., excellent, good, fair, poor) [33,34]. Study methodological quality will be evaluated per measurement property and determined by the lowest checklist rating [33,34]. Two reviewers will independently undertake data extraction and apply the checklist to each included study. Consensus will be sought through discussion; any disagreements will be resolved using a third reviewer.

\section{Data synthesis}

As reported in other reviews [37,38], all data will be qualitatively synthesised to determine the overall quality and acceptability of each reviewed measure. The synthesis will take the following factors into account: i) study methodological quality (COSMIN scores); ii) the number of studies reporting specific evidence per measure; iii) the results for each measurement/practical property per measure; and iv) consistency between studies [38]. The data synthesis score will have two elements. First, the overall quality of a measurement property will be reported as: adequate $(+)$, not adequate $(-)$, conflicting $(+/-)$, or unclear (?). Second, levels of evidence for the overall quality of each measurement property will be further defined to indicate 'strong, 'moderate', 'limited', 'conflicting', or 'unknown' evidence as detailed by Elbers et al. [38]. The synthesis will produce a shortlist of 'best' quality measures that will be further considered in stage two.

\section{Stage 2: Consensus meeting}

The final stage of the project will be a one-day 'expert' consensus meeting with 15 participants representing key stakeholders, including lay representatives $(n=6)$, clinicians who use therapeutic exercise $(n=3)$, expert researchers in the field of adherence or exercise therapy $(\mathrm{n}=3)$, and service managers $(\mathrm{n}=3)$. We will reach a consensus on the 'best' measures of exercise adherence in terms of quality, relevance, acceptability, feasibility, and appropriateness for musculoskeletal healthcare in both clinical research and routine practice settings. The strengths and limitations of reviewed measures, and a future research agenda will be produced.

A structured group decision-making approach, or Nominal Group Technique [39-41], will be used to work towards consensus on two main questions:

1. What should be measured when assessing exercise adherence?

2. How should exercise adherence be measured and how 'useful' are the shortlisted 'best' measures of exercise adherence with respect to:

- Relevance (to the aspects of exercise adherence viewed as most important);

- Acceptability (to patients who are required to adhere to exercise regimes);

- Appropriateness (to the musculoskeletal population)

- Feasibility (for use in clinical research and/or routine practice settings)?

Relevant research will be summarised and sent to participants in advance of the meeting. This will include i) a synthesis of the systematic review and copies of the 'shortlisted' measures (how is adherence currently measured); and ii) a list of aspects, or domains, of exercise adherence currently assessed in published research (what aspects of adherence are currently measured).

Participants will also receive a questionnaire with two key sections on what and how to measure exercise adherence. Participants will be asked to rate the relative importance of each aspect, or domain of exercise adherence identified in the systematic review. Importance ratings will be made on a 9-point GRADE scale (1 to 3 $=$ not important; 4 to $6=$ important; 7 to $9=$ critical) $[42,43]$. Importance will be defined as 'how important is it that this aspect, or domain, of adherence is included in the assessment of exercise adherence?' Participants will also be asked to consider the relevance and feasibility of each measure for research or clinical musculoskeletal settings. Measures will be rated on separate 9-point GRADE scales ( 1 to $3=$ not relevant/not feasible; 4 to $6=$ relevant/feasible; 7 to $9=$ most relevant/highly feasible). Finally, participants will be asked to rate the suitability of each measure for the assessment of exercise adherence in i) research (yes/no) or ii) routine practice (yes/no) musculoskeletal settings.

The questionnaire content will be piloted with representative stakeholders $(\mathrm{n}=3$; to include a patient, physiotherapist, methodologist/researcher). Participants will be asked to return all completed questionnaires in 
advance of the meeting to allow results to be collated. Where appropriate, participants will be encouraged to provide additional comments or contributions to the candidate lists of domains and outcome measures for further discussion at the meeting.

The consensus meeting will be structured into three discrete sections [44-46]. First the evidence synthesis will be re-presented [43], and group results from postal completion of the nominal group questionnaire shared with the group. Individual participants will also receive their own individual scores, supporting comparison with the wider group. Next, semi-structured group discussions will be facilitated and participants will again be invited to address the two core questions stated above. Finally, a plenary session will be convened and the results from the group sessions fed back to all participants. There will be an anonymised voting process, during which we will converge on a common view of what aspects of exercise adherence should be assessed (1. What to measure?), and make recommendations for the most relevant and appropriate method of assessment (2. How to measure?). During the final vote participants will be invited to vote as to whether each domain (yes/no) and each outcome measure (yes/no) should be included in the assessment of exercise adherence. The meeting will seek to make clear recommendations for simple, relevant, and appropriate assessment of exercise adherence both within research and clinical practice settings within the musculoskeletal field.

\section{Discussion}

Adherence to therapeutic exercise is a pre-requisite for successful rehabilitation, yet its accurate measurement is challenging [11]. In clinical practice, more objective measures of exercise adherence are underutilised [28]. In clinical trials of exercise for musculoskeletal pain, adherence is not always measured, and when it is, the measures used are often not validated or standardised, making it difficult to compare the effectiveness of different interventions and impossible to pool data in the form of a meta-analyses [7,29]. This review will identify, summarise, and critically evaluate available measures of exercise adherence and develop recommendations about the most promising measures which are relevant for musculoskeletal research and clinical practice. This will benefit clinicians evaluating patients' levels of exercise adherence and those intending to undertake research, service evaluation, or audit relating to exercise adherence in the musculoskeletal field. The findings will impact upon new research studies which aim to understand the factors that predict adherence with exercise, which test different adherence-enhancing interventions.

\section{Abbreviations}

AMED: Allied and complementary medicine; CINAHL: Cumulative index to nursing and allied health literature; COSMIN: COnsensus-based Standards for the selection of health Measurement Instruments; GRADE: Grading of recommendations assessment development and evaluation; PRISMA: Preferred reporting items for systematic reviews and meta-analyses; UK: United Kingdom.

\section{Competing interests}

The authors declare that they have no competing interests.

\section{Authors' contributions}

All authors have made substantial contributions to the conception and design of the study and participated in writing the grant application. MH drafted the manuscript. SM, KH, TP, and MG edited and finalised the manuscript. All authors read and approved the final manuscript.

\section{Acknowledgements}

We are grateful for the involvement of the Project Steering Group (Hazel Horobin, Devdeep Ahuja, and Jeff Breckon) and patient representatives from Barnsley Consumer Research Advisory Group for their contribution to the development of this project. The Chartered Society of Physiotherapy Charitable Trust funded this research.

\section{Author details}

${ }^{1}$ Arthritis Research UK Primary Care Centre, Keele University, Keele ST5 5BG, UK. ${ }^{2}$ Royal College of Nursing Research Institute, Warwick Medical School, Warwick University, Coventry CV4 7AL, UK. ${ }^{3}$ Faculty of Health and Wellbeing, Collegiate Campus, Sheffield Hallam University, Sheffield S10 2BP, UK. ${ }^{4}$ Centre for Health and Social Care Research, Collegiate Campus, Sheffield Hallam University, Sheffield S10 2BP, UK.

Received: 20 January 2014 Accepted: 28 January 2014

Published: 10 February 2014

\section{References}

1. Parsons $S$, Breen A, Foster NE, Letley L, Pincus T, Vogel S, Underwood M: Prevalence and comparative troublesomeness by age of musculoskeletal pain in different body locations. Fam Pract 2007, 24:308-316.

2. Reginster JY: The prevalence and burden of arthritis. Rheumatology 2002, 41(Suppl 1):3-6.

3. Woolf AD, Pfleger B: Burden of major musculoskeletal conditions. Bull World Health Organisat 2003, 81:646-656.

4. Walsh NE, Brooks P, Hazes JM, Walsh RM, Dreinhofer K, Woolf AD, Akesson K, Lidgren L, Bone and Joint Decade Task Force for Standards of Care for Acute and Chronic Musculoskeletal Pain: Standards of care for acute and chronic musculoskeletal pain: the bone and joint decade (2000-2010). Arch Phys Med Rehabil 2008, 89:1830-1845.

5. Childs JD, Cleland JA, Elliott JM, Teyhen DS, Wainner RS, Whitman JM, Sopky BJ, Godges JJ, Flynn TW, American Physical Therapy Association: Neck pain: clinical practice guidelines linked to the international classification of functioning, disability, and health from the orthopaedic section of the American physical therapy association. J Orthop Sports Phys Ther 2008, 38:A1-A34.

6. Fernandes L, Hagen KB, Bijlsma JW, Andreassen O, Christensen P, Conaghan PG, Doherty M, Geenen R, Hammond A, Kjeken I, Lohmander LS, Lund H, Mallen CD, Nava T, Oliver S, Pavelka K, Pitsillidou I, da Silva JA, de la Torre J, Zanoli G, Vliet Vlieland TP, European League Against Rheumatism (EULAR): EULAR recommendations for the non-pharmacological core management of hip and knee osteoarthritis. Ann Rheum Dis 2013, 72(7):1125-1135.

7. Jordan $J$, Holden ME, Mason EEJ, Foster NE: Interventions to improve adherence to exercise for chronic musculoskeletal pain in adults. Cochrane Database Syst Rev 2010, 1, CD005956.

8. Fuentes JP, Armijo-Olivo S, Magee DJ, Gross DP: Effects of exercise therapy on endogenous pain-relieving peptides in musculoskeletal pain - a systematic review. Clin J Pain 2011, 27:365-374.

9. Kay TM, Gross A, Goldsmith C, Santaguida PL, Hoving J, Bronfort G, Cervical Overview Group: Exercises for mechanical neck disorders. Cochrane Database Syst Rev 2005, 3, CD004250.

10. Fransen M, McConnell S: Exercise for osteoarthritis of the knee. Cochrane Database Syst Rev 2008, 4, CD004376.

11. World Health Organisation: Adherence to Long Term Therapies - Evidence for Action. Geneva: World Health Organisation; 2003. 
12. Roddy E, Zhang W, Doherty M, Arden KM, Barlow J, Birrell F, Carr A, Chakravarty K, Dickson J, Hay E, Hosie G, Hurley M, Jordan KM, McCarthy C, McMurdo M, Mockett S, O'Reilly S, Peat G, Pendleton A, Richards S: Evidence-based recommendations for the role of exercise in the management of osteoarthritis of the hip or knee-the MOVE consensus. Rheumatology 2005, 44:67-73.

13. McLean SM, May S, Klaber Moffett J, Sharp D, Gardiner E: Prognostic factors for progressive non-specific neck pain. Phys Ther Rev 2007, 12:207-220.

14. Hayden JA, van Tulder MW, Tomlinson G: Systematic review: strategies for using exercise therapy to improve outcomes in chronic low back pain. Ann Intern Med 2005, 142:776-785.

15. McLean S, Klaber Moffett J, Sharp D, Gardiner E: A randomised controlled trial comparing graded exercise treatment and usual physiotherapy for patients with non-specific neck pain (The GET UP neck pain trial). Man Ther 2013, 18:199-205.

16. Kolt GS, McEvoy JF: Adherence to rehabilitation in patients with low back pain. Man Ther 2003, 8:110-116.

17. Forkan R, Pumper B, Smyth N, Wirkkala H, Ciol MA, Shumway-Cook A: Exercise adherence following physical therapy intervention in older adults with impaired balance. Phys Ther 2006, 86:401-410.

18. Hardage J, Peel C, Morris D, Graham C, Brown C, Foushee HR, Braswell J: Adherence to exercise scale for older patients (AESOP): a measure for predicting exercise adherence in older adults after discharge from home health physical therapy. J Geriatr Phys Ther 2007, 30:69-78.

19. Gucciardi E: A systematic review of attrition from diabetes education services: strategies to improve attrition and retention research. Can J Diabetes 2008, 32:53-65.

20. Martin C, Perfect T, Mantle G: Non-attendance in primary care: the views of patients and practices on its causes, impact and solutions. Fam Pract 2005, 22:638-643.

21. Weinger K, McMurrich SJ, Yi JP, Lin S, Rodriguez M: Psychological characteristics of frequent short-notice cancellers of diabetes medical and education appointments. Diabetes Care 2005, 28:1791-1793.

22. Turk DC, Rudy TE: Neglected topics in the treatment of chronic pain patients-relapse, noncompliance, and adherence enhancement. Pain 1991, 44:5-28.

23. Beswick AD, Rees K, West RR, Taylor FC, Burke M, Griebsch I, Taylor RS, Victory J, Brown J, Ebrahim S: Improving uptake and adherence in cardiac rehabilitation: literature review. J Adv Nurs 2005, 49:538-555.

24. Wens J, Vermeire $E_{1}$ Hearnshaw $H$, Lindenmeyer A, Biot $Y$, Van Royen $P$ : Educational interventions aiming at improving adherence to treatment recommendations in type 2 diabetes: a sub-analysis of a systematic review of randomised controlled trials. Diabetes Res Clin Pract 2008, 79:377-388

25. Williams A, Manias E, Walker R: Interventions to improve medication adherence in people with multiple chronic conditions: a systematic review. J Adv Nurs 2008, 63:132-143.

26. Kolt GS, Brewer BW, Pizzari T, Schoo AMM, Garrett N: The sport injury rehabilitation adherence scale: a reliable scale for use in clinical physiotherapy. Physiotherapy 2007, 93:17-22.

27. Treuth MS: Applying multiple methods to improve the accuracy of activity assessments. In Physical Activity Assessments for Heath Related Research. Edited by Welk GJ. USA: Human Kinetics Publishers; 2002:213-224.

28. Holden MA, Nicholls EE, Hay EM, Foster NE: Physical therapists' use of therapeutic exercise for patients with clinical knee osteoarthritis in the United Kingdom: in line with current recommendations? Phys Ther 2008 88:1109-1121.

29. Beinart NA, Goodchild CE, Weinman JA, Ayis S, Godfrey EL: Individual and intervention-related factors associated with adherence to home exercise in chronic low back pain: a systematic review. Spine J 2013, 13:1940-1950.

30. Abrams D, Davidson M, Harrick J, Harcourt P, Zylinski M, Clancy J: Monitoring the change: current trends in outcome measure usage in physiotherapy. Man Ther 2006, 11:46-53.

31. Ward JA, Potter S, Blazeby JM: Outcome reporting for reconstructive breast surgery: the need for consensus, consistency and core outcome sets. Eur J Surg Oncol 2012, 38:1020-1021.

32. The PRISMA group: Preferred Reporting Items for Systematic reviews and MetaAnalyses: The PRISMA statement. 2009. http://www.prisma-statement.org/.

33. Terwee CB, Mokkink LB, Knol DL, Ostelo RW, Bouter LM, de Vet HC: Rating the methodological quality in systematic reviews of studies on measurement properties: a scoring system for the COSMIN checklist. Qual Life Res 2012, 21:651-657.

34. Mokkink LB, Terwee CB, Patrick DL, Alonso J, Stratford PW, Knol DL, Bouter LM, de Vet HC: The COSMIN study reached international consensus on taxonomy, terminology, and definitions of measurement properties for health-related quality patient-reported outcomes. J Clin Epidemiol 2010, 63:737-745

35. Terwee CB, Jansma EP, Riphagen II, de Vet HCW: Development of a methodological PubMed search filter for finding studies on measurement properties of measurement instruments. Qual Life Res 2009, 18:1115-1123.

36. Haywood KL, Staniszewska S, Chapman S: Quality and acceptability of patient-reported outcome measures used in chronic fatigue syndrome/ myalgic encephalomyelitis (CFS/ME): a systematic review. Qual Life Res 2012, 21:35-52.

37. Haywood KLBJ, Tutton E, Staniszewska S: Patient-reported outcome measures (PROMs) and fragility hip fracture: a systematic review of quality and acceptability. Qual Life Res 2013, 22(Suppl 1):2077.

38. Elbers RG, Rietberg MB, van Wegen EE, Verhoef J, Kramer SF, Terwee CB, Kwakkel G: Self-report fatigue questionnaires in multiple sclerosis, Parkinson's disease and stroke: a systematic review of measurement properties. Qual Life Res 2012, 21:925-944.

39. Gallagher M, Hares T, Spencer J, Bradshaw C, Webb I: The nominal group technique: a research tool for general practice? Fam Pract 1993, 10:76-81.

40. Harvey $N$, Holmes CA: Nominal group technique: an effective method for obtaining group consensus. Int J Nurs Pract 2012, 18:188-194.

41. Foster NE, Dziedzic KS, van der Windt D, Fritz J, Hay EM: Research priorities for non-pharmacological therapies for common musculoskeletal problems: nationally and internationally agreed recommendations. BMC Musculoskelet Disord 2009, 10:3.

42. Grade Working Group. http://www.gradeworkinggroup.org.

43. Williamson PR, Altman DG, Blazeby JM, Clarke M, Devance D, Gargon E, Tugwell P: Developing core outcome sets for clinical trials: issues to consider. Trials 2012, 13:132.

44. Murphy MK, Black NA, Lamping DL, McKee CM, Sanderson CF, Askham J, Marteau T: Consensus development methods, and their use in clinical guideline development. Health Technol Assess 1998, 2(3):1-88. i-iv.

45. Vella K, Goldfrad C, Rowan K, Bion J, Black N: Use of consensus development to establish national research priorities in critical care. BMJ 2000, 320:976-980.

46. Sarre G, Cooke J: Developing indicators for measuring research capacity development in primary care organizations: a consensus approach using a nominal group technique. Health Soc Care Community 2009, 17:244-253.

doi:10.1186/2046-4053-3-10

Cite this article as: Holden et al:: Recommendations for exercise adherence measures in musculoskeletal settings: a systematic review and consensus meeting (protocol). Systematic Reviews 2014 3:10.

\section{Submit your next manuscript to BioMed Central and take full advantage of:}

- Convenient online submission

- Thorough peer review

- No space constraints or color figure charges

- Immediate publication on acceptance

- Inclusion in PubMed, CAS, Scopus and Google Scholar

- Research which is freely available for redistribution 